\title{
Vocal Characteristics and Laryngoscopic Findings in Future Musical Theater Performers
}

\author{
*Evelien D’haeseleer, +Sofie Claeys, *Iris Meerschman, *Kim Bettens, *Sofie Degeest, \\ *Caroline Dijckmans, *Joke De Smet, *Anke Luyten, and *,‡Kristiane Van Lierde \\ *Department of Speech, Language and Hearing Sciences, Ghent University, Ghent, Belgium; \\ tDepartment of Otorhinolaryngology and Head and Neck Surgery, Ghent University Hospital, Ghent, Belgium; \\ ‡Department of Speech-Language Pathology and Audiology, University of Pretoria, Pretoria, South Africa.
}

Address correspondence to Evelien D'haeseleer, Department of Speech, Language and Hearing Sciences, Ghent University, De Pintelaan 185, 2P1, 9000 Ghent, Belgium. E-mail: Evelien.Dhaeseleer@ugent.be

This work was presented at the 44th Annual Symposium of the Voice Foundation in 2015 (D'haeseleer E., De Ley S., Claeys S., Bettens K., Degeest S., Luyten A., Dijckmans C., De Smet J., Van Lierde K. [2015]. Voice Quality in Future Musical Actors. 44th Annual Symposium: Care of the professional voice, Philadelphia, May 29-June 2, 2015, Poster presentation) and at the 45th Annual Symposium of the Voice Foundation in 2016 (D'haeseleer E., Meerschman, I. Claeys S., Bettens K., Degeest S., Luyten A., Dijckmans C., De Smet J., Kristiane Van Lierde K. Voice symptoms and risk factors for developing voice disorders in future musical actors. 45th Annual Symposium: Care of the professional voice, Philadelphia, June 1-5, 2016, Oral presentation).

\section{Summary}

Objective: Musical theater performers are a special group of elite vocal performers with a high vocal load as they combine singing, acting, and physical performance. As they are absolutely depending on their voice quality and vocal capacities for their studies and their future profession, an optimal voice production is very important. The purpose of this study was to determine the voice quality of musical theater students. The voice quality of seven students was then reevaluated 1 year after the first assessment.

Study design: Observational study.

Methods: Thirty-one musical students ( 7 men and 24 women) with a mean age of 20 years participated in the study. To determine the voice quality, objective (aerodynamic measurements, voice range profile, acoustic analysis, and Dysphonia Severity Index) and subjective (videolaryngostroboscopy, Voice Handicap Indexes, and questionnaires regarding voice symptoms and risk factors) voice measurements were performed.

Results: The median Dysphonia Severity Index in male and female musical students was respectively 5.3 and 5.7, both corresponding with an overall good voice quality. The questionnaires revealed the presence of vocal fatigue, dryness of the throat, vocal tract discomfort, and harmful vocal habits in the majority of students. In $45 \%$ of the subjects, videolaryngostroboscopic evaluation of the vocal folds showed an organic lesion. The majority of these lesions are inflammatory lesions (26\%). In $68 \%$ of the subjects, a certain degree of supraglottic constriction was observed.

Conclusion: Despite the overall good voice quality, videolaryngostroboscopy showed a high presence of vocal fold lesions and supraglottic constriction during phonation. 
Key Words: Musical theater actors; Future elite vocal performers; Vocal characteristics; Vocal fold function; Vocal quality

\section{Introduction}

In elite vocal performers, like singers and actors, even a slight vocal difficulty can have serious professional consequences. ${ }^{1}$ Even within this group of voice users, different voice use and vocal demands are described per profession. ${ }^{2}$ Actors must be able to adjust their voice production to theaters of varying size while maintaining the ability to express the full range of human emotion. ${ }^{3}$ Their performance is sometimes characterized by extreme physical exertions on stage and sudden emotional outbursts, such as screaming, shouting, crying, or sobbing. ${ }^{4}$ Roy et al $^{4}$ describe this as vocally violent behavior. Singing is associated with other typical vocal demands. Singers use a breathy or "rough" type of voice or they sing with a specific balance of tonality depending on the type of singer (pop/rock versus classically trained). ${ }^{2}$ Musical theater performers are a special group of elite vocal performers with a high vocal load as they combine singing, acting, and physical performance (dancing). They typically sing during a highly exhausting choreographed production and combine it with acting, including the "vocally violent behavior." This unique performance genre, typically consisting of eight shows a week, requires heavy vocal demands. ${ }^{5}$

Environment plays an important role in voice performance. Off-stage behavior in performers is sometimes characterized by poor vocal hygiene as they do not always employ enough vocal and physical rest. ${ }^{6}$ Hoffman-Ruddy et $\mathrm{al}^{2}$ described that the "stage environment" also influences voice production of musical theater performers. Sometimes theaters where the shows and the rehearsals take place can be dusty. ${ }^{3}$ On stage, smoke during the performance can also affect the vocal quality. Another environmental factor typical for musical theater performers are the costumes and prosthetic devices they wear during performing and singing. Sometimes costumes can be extremely warm, heavy, or restricting. ${ }^{3}$ Finally, makeup limiting facial mobility can make projection and articulation more difficult. ${ }^{2}$ It is clear that vocal performance in musical theater requires extremely high vocal demand. ${ }^{5}$ As they are absolutely depending on their vocal quality and vocal capacities for their profession, an optimal voice production is very important. Consequently, voice screenings of these voice athletes are necessary and should also be implemented in the programs of music and performing arts. ${ }^{7}$ Musical theater students receive an intensive training in singing, acting, and dancing (about 8 hours per day), and are expected to participate in long rehearsals and full performances. They are considered as a high-risk population for developing voice problems (such as overuse injuries) because of the heavy vocal load. ${ }^{5 ; 6 ;} 7$ However, limited data exist regarding the baseline of vocal symptoms, vocal habits, and vocal quality in this population. Timmermans et al $^{6}$ described reduced vocal quality in future elite vocal performers. Donahue et $\mathrm{al}^{7}$ investigated the vocal symptoms and habits of musical theater students and found that the majority noted poor hydration habits and more than $50 \%$ of the students reported at least one current negative vocal symptom. They recommended more education regarding vocal hygiene in the group of musical theater students. 
The purpose of this study was to investigate the anatomy and physiology of the vocal folds, vocal quality of the speaking voice, voice symptoms, and vocal habits in 31 musical theater students using a combination of subjective (videolaryngostroboscopy for the evaluation of the vocal folds and the vocal function, perceptual evaluation of the voice, Voice Handicap Index [VHI], and questionnaires investigating voice symptoms and risk factors for developing voice problems) and objective (aerodynamic measurements, vocal range, acoustic analysis, and Dysphonia Severity Index [DSI]) voice measurements. The vocal quality of seven students was then reevaluated 1 year after the first assessment. Hypothetically, the vocal quality of future elite vocal performers is excellent and voice capacities improve during their studies in music and performing arts. The study will provide a database of vocal characteristics and habits of musical theater students that can be used as a reference for future students. A better understanding of their vocal habits will help improve vocal coaching and prevent voice problems.

\section{Methods}

\section{Subjects}

Thirty-one students from a bachelor program in music and performing arts (musical), 7 men and 24 women with a mean age of 20.7 years (SD: 1.89), participated in the study. Inclusion criteria were full-time student (male or female) in the first or second year of the musical program, good physical and mental states of well-being, and normal hearing. Twenty-three students were recruited from the first bachelor year and eight students were recruited from the second bachelor year of the program. All students succeeded in the entrance test of the musical program consisting of a dancing, singing, and acting test. The entrance test for being admitted to the program was a test of potential rather than ability and did not include a medical voice screening.

The musical theater students underwent the voice assessment protocol. Otoscopy and audiometry were performed by an experienced audiologist (S.D.). All students had normal hearing corresponding with pure tone average less than $20 \mathrm{~dB}$ in the poorer ear. Vocal load in the bachelor program musical is very high. Intensive courses in singing and acting each day are followed. The time of singing per day varies between 1 and 3 hours (mean: 1.6 hours). Acting classes and rehearsals vary between 1 and 6 hours (mean 3.1 hours) per day. Characteristics of voice use and misuse are presented in Table 1, Table 2 ; Table 3.

Table 1. Results of the Voice Handicap Index (VHI) and the VHI Adapted to the Singing Voice in Musical Theater Students

\begin{tabular}{|c|c|c|c|c|c|c|c|c|c|c|}
\hline & & & Men & & & & & Women & & \\
\hline & Mean & Median & Minimum & Maximum & SD & Mean & Median & Minimum & Maximum & SD \\
\hline VHI & 7.1 & 5.0 & 2.0 & 24.0 & 7.8 & 14.0 & 10.0 & 0.0 & 60.0 & 13.7 \\
\hline VHI singing voice & 14.3 & 9.0 & 5.0 & 30.0 & 10.5 & 20.3 & 15.0 & 1.0 & 49.0 & 12.7 \\
\hline
\end{tabular}


Table 2. Reported Voice Symptoms in Musical Theater Students

\begin{tabular}{|c|c|c|c|c|c|c|c|c|}
\hline \multirow{2}{*}{ Voice Symptoms } & \multicolumn{2}{|c|}{ Never } & \multicolumn{2}{|c|}{ Daily } & \multicolumn{2}{|c|}{ Weekly } & \multicolumn{2}{|c|}{ Monthly } \\
\hline & $\mathbf{n}$ & $\%$ & $\mathbf{n}$ & $\%$ & $\mathbf{n}$ & $\%$ & $\mathbf{n}$ & $\%$ \\
\hline Vocal tract discomfort & 8 & 26.7 & 2 & 6.7 & 4 & 13.3 & 16 & 53.3 \\
\hline Vocal fatigue & 6 & 20.0 & 2 & 6.7 & 9 & 30.0 & 13 & 43.3 \\
\hline Irritation or pain & 14 & 46.7 & 2 & 6.7 & 4 & 13.3 & 10 & 33.3 \\
\hline Dryness of the throat & 6 & 20.0 & 2 & 6.7 & 8 & 26.7 & 14 & 46.7 \\
\hline Pain & 18 & 60.0 & 3 & 10.0 & 1 & 3.3 & 8 & 26.7 \\
\hline Tickling feeling & 14 & 46.7 & 2 & 6.7 & 3 & 10.0 & 11 & 36.7 \\
\hline Feeling of throat tightness and pressure in the throat & 17 & 58.6 & 0 & 0.0 & 6 & 20.7 & 6 & 20.7 \\
\hline Burning feeling in the throat & 20 & 66.7 & 1 & 3.3 & 0 & 0.0 & 9 & 30.0 \\
\hline Lump in throat feeling & 16 & 53.3 & 1 & 3.3 & 2 & 6.7 & 11 & 36.7 \\
\hline Hoarse voice & 21 & 70.0 & 1 & 3.3 & 2 & 6.7 & 6 & 20.0 \\
\hline Cracking voice & 19 & 63.3 & 1 & 3.3 & 2 & 6.7 & 8 & 26.7 \\
\hline Voice instability & 24 & 80.0 & 2 & 6.7 & 0 & 0.0 & 4 & 13.3 \\
\hline Changes in pitch & 26 & 86.7 & 1 & 3.3 & 0 & 0.0 & 3 & 10.0 \\
\hline Reduced pitch range & 20 & 69.0 & 2 & 6.9 & 0 & 0.0 & 7 & 24.1 \\
\hline Difficulties in voice projection & 23 & 76.7 & 1 & 3.3 & 0 & 0.0 & 6 & 20.0 \\
\hline Feeling that talking is tiring & 24 & 80.0 & 0 & 0.0 & 2 & 6.7 & 4 & 13.3 \\
\hline Get out of breath when talking & 25 & 83.3 & 0 & 0.0 & 1 & 3.3 & 4 & 13.3 \\
\hline Only be able to whisper & 30 & $\begin{array}{c}100 . \\
0\end{array}$ & 0 & 0.0 & 0 & 0.0 & 0 & 0.0 \\
\hline
\end{tabular}

Table 3. Presence of Risk Factors for Developing Voice Disorders in Musical Theater Students

Using a tensed voice

Frequently coughing

Frequently throat clearing

Speaking during a laryngitis

Hormonal imbalance

Allergies/sinusitis

Frequent colds and flu

Reflux

Alcohol, nicotine, recreational drugs

Shouting above a background noise

Habitual loud voice use

Shouting/screaming

Screaming over a distance

Singing and acting with a poor technique

Using the voice with an inappropriate pitch

Stress/anxiety

Use of unnatural intonation patterns

\begin{tabular}{|c|c|c|c|c|c|c|c|}
\hline \multicolumn{2}{|c|}{ Never } & \multicolumn{2}{|c|}{ Daily } & \multicolumn{2}{|c|}{ Weekly } & \multicolumn{2}{|c|}{ Monthly } \\
\hline$n$ & $\%$ & $n$ & $\%$ & $n$ & $\%$ & $n$ & $\%$ \\
\hline 13 & 43.30 & 0 & 0.00 & 5 & 16.70 & 12 & 40.00 \\
\hline 17 & 56.70 & 3 & 10.00 & 1 & 0.50 & 9 & 30.00 \\
\hline 14 & 46.70 & 4 & 13.30 & 6 & 20.00 & 6 & 20.00 \\
\hline 22 & 73.30 & 1 & & 0 & 0.00 & 7 & 23.30 \\
\hline 22 & 73.30 & 2 & 0.10 & 0 & 0.00 & 6 & 20.00 \\
\hline 20 & 66.70 & 3 & 10.00 & 1 & ( & 6 & 20.00 \\
\hline 22 & 73.30 & 3 & 10.00 & 0 & 0.00 & 5 & 16.70 \\
\hline 24 & 80.00 & 1 & 3.30 & 3 & 10.00 & 2 & 6.70 \\
\hline 16 & 53.30 & 4 & 13.30 & 5 & 16.70 & 5 & 16.70 \\
\hline 10 & 33.30 & 2 & 6.70 & 10 & 33.30 & 8 & 26.70 \\
\hline 17 & 56.70 & 4 & 13.30 & 6 & 20.00 & 3 & 10.00 \\
\hline 9 & 30.00 & 2 & 6.70 & 10 & 33.3 & 9 & 30.00 \\
\hline 17 & 56.70 & 3 & 10.00 & 3 & 10.00 & 7 & 23.30 \\
\hline 19 & 63.30 & 1 & 3.30 & 5 & 16.70 & 5 & 16.70 \\
\hline 24 & 80.00 & 1 & 3.30 & 2 & 6.70 & 3 & 10.00 \\
\hline 4 & 13.30 & 2 & 6.70 & 8 & 26.70 & 16 & 53.30 \\
\hline 27 & 90.00 & 0 & 0.00 & 1 & 3.30 & 2 & 6.70 \\
\hline
\end{tabular}


To determine the vocal quality of the subjects, a combination of objective (aerodynamic measurements, voice range, acoustic analysis, DSI) and subjective (perceptual evaluation, videolaryngostroboscopy, self-assessment using Voice Handicap Index, and questionnaires investigating voice symptoms and risk factors for developing voice problems) assessment techniques were used.

\section{Questionnaires}

The self-perception of the impact of vocal problems on the psychosocial functioning was measured using the Dutch version ${ }^{8}$ of the $\mathrm{VHI} .{ }^{9}$ For the subject's perception of the singing voice, the Dutch version ${ }^{10}$ of the VHI adapted to the singing voice ${ }^{11}$ was used. The VHI and the $\mathrm{VHI}$ adapted to the singing voice are self-administered questionnaires consisting of 30 statements. Each statement is evaluated on a 4-point grading scale $(0=$ never, $1=$ almost never, 2 = sometimes, 3 = almost always, 4 = always). The total $\mathrm{VHI}$ score varies between 0 and 120 and the higher the score, the more perceived disability due to voice difficulties of respectively the speaking and the singing voice is present.

To inventory the voice symptoms and the risk factors for developing voice disorders, the Dutch version of the checklists ${ }^{12}$ of Russel et al $^{13}$ were used. The questionnaires investigate the presence and the frequency (never, daily, weekly, monthly) of voice symptoms and risk factors (vocal abuse, vocal misuse) ${ }^{13}$ as reported by the subjects.

\section{Videolaryngostroboscopy}

Each subject underwent a videolaryngostroboscopy performed by an ENT specialist (S.C.) with more than 20 years of experience in voice disorders. The subjects were asked to produce the sustained vowel /i/ at habitual pitch and loudness followed by a glissando. A standardized evaluation protocol ${ }^{14}$ was used for all musical students to evaluate the aspect and the function of the vocal folds during habitual voice production. The following characteristics of the videolaryngostroboscopic assessment were evaluated: symmetry (symmetrical or asymmetrical), regularity (regular, irregular, or inconsistent), glottic closure (complete, incomplete, and inconsistent), type of gap (longitudinal, posterior, anterior, irregular, oval, and hourglass), amplitude (increased, normal, reduced, and none), and mucosal wave (normal, reduced, and none). Secondly, the aspect of the vocal folds (normal, organic lesion, and color) was evaluated. The presence of possible supraglottic contraction was observed in two directions, mediolateral (M-L) and anterior-posterior (A-P), using the SERF protocol. ${ }^{15}$ The SERF protocol pictures a laryngeal image with laryngeal concentric circles. The constrictions were evaluated using a 4-point grading scale $(0=$ no constriction, $5=$ very severe constriction). The same voice therapist (E.D.) completed all evaluations of the randomized samples. An interobserver reliability was used. Therefore, $20 \%$ of the samples were judged at random, blinded and independently by another voice therapist (I.M.).

\section{Perceptual evaluation}

For the auditory-perceptual rating of the voices during connected speech, the GRBAS scale from Hirano ${ }^{16}$ was used. This is the most widely used scale for auditory-perceptual 
evaluation recommended by the Japan Society of Logopedics and Phoniatrics. The GRBAS scale contain five parametersdescribing different aspects of vocal quality: $G$ (overall grade of hoarseness), R (roughness), B (breathiness), A (asthenic quality), and S (strained quality) of the voice. Each parameter was scored using a 4-point grading scale $(0=$ normal, $1=$ slight, $2=$ moderate, and $3=$ severe). A sixth parameter $I^{17}$ for instability of the voice was added to the GRBAS scale. The subjects were asked to read aloud a Dutch text (The north wind and the sun). The voice samples of connected speech during reading were audio recorded using a microphone (CO1U USB Studio Condenser Microphone, Samson) and the software program PRAAT (www.PRAAT.org). The samples were rated blinded and at random by the same voice therapist (E.D.) with ample experience in voice and voice disorders. An interobserver reliability was used. Twenty percent of the voice samples were selected at random and rated blinded and independently by another voice therapist (C.D.).

\section{Objective voice assessment}

The objective voice assessment consists of the determination of aerodynamic, vocal range, and acoustic parameters and DSI measurements.

The following aerodynamic parameters were determined: the maximal phonation time (MPT), the vital capacity (VC), and the phonation quotient. For the MPT, the subjects were instructed to produce the sustained vowel /a/ at a habitual pitch and loudness after a maximal inspiration. The length of the sustained phonation was measured with a chronometer (C510-B; Oregon Scientific, Berkshire, UK). For the determination of the VC, a dry spirometer (Riester, Jungingen, Germany) was used. The experimenter functioned as a model and provided verbal encouragement. The best value of three attempts was retained. The phonation quotient is the ratio of the VC to the MPT.

The vocal range of the subjects in terms of the highest and lowest frequency and intensity was measured using the Voice Range Profile (VRP) of the Computerized Speech Lab of Kay Elemetrics. The procedure described by Heylen et $\mathrm{al}^{18}$ was used. The patients were instructed to phonate the vowel /a/ for at least 2 seconds and with an acceptable vocal quality (as judged by the experimenter) at a habitual pitch and loudness, the lowest pitch (Flow), the lowest intensity (llow), the highest frequency (Fhigh), and the highest intensity (Ihigh).

An acoustic analysis of the sustained vowel /a/ was performed using the Multidimensional Voice Program of the Computerized Speech Lab. The following parameters were determined: mean fundamental frequency $\left(F_{0}\right.$ in $\left.\mathrm{Hz}\right)$, jitter (\%), shimmer (\%), variation of $\mathrm{F}_{0}$ $\left(v F_{0}\right)$, and noise-to-harmonic ratio. The subjects were instructed to produce the vowel /a/ using a comfortable pitch and intensity. A midvowel segment from 3 seconds registered with a sampling rate from $50,000 \mathrm{~Hz}$ was used.

The DSI ${ }^{19}$ is a multiparameter approach to evaluate the objective vocal quality. The index is based on a weighted combination of the above-described parameters: MPT, jitter (\%), Fhigh, and llow. The DSI varies between -5 and +5 for, respectively, severely dysphonic vocal quality and good vocal quality. Values higher than 5 are possible in subjects with very good vocal capacities. The cutting point between normal and dysphonic voices is $1.6 .{ }^{20}$ 


\section{Statistical analysis}

SPSS version 22 (SPSS Corporation, Chicago, IL) was used for the statistical analysis of the data. For the comparison of the data of the first assessment with the follow-up assessment (1 year later), the paired Wilcoxon test (nonparametric data) was applied. The chi-square test was used to compare the data of the videolaryngostroboscopy of both assessments.

\section{Results}

\section{Voice characteristics of musical theater students}

\section{Questionnaires}

The results of the $\mathrm{VHI}$ and the $\mathrm{VHI}$ adapted to the singing voice are presented in Table 1. Table 2 provides an overview of the reported voice symptoms and their frequency of occurrence (never, daily, weekly, monthly). In Table 3, the presence of risk factors for developing voice disorders in the group of musical students is described and Table 4 provides an overview of the different behaviors of vocal abuse.

Table 4. Presence of Vocal Abuse in Musical Theater Students

Coughing and throat clearing
Whispering
Yelling and screaming
Imitation of voices
Growling
Imitation of sounds
Inhalation phonation
Using hard voice onsets
Using an inappropriate vocal volume
Using a high pitch
Using a high speech rate
Using a tensed voice
Often speaking to large groups
Speaking with irregular breathing or breaks
Speaking without a break (more than 1 hour)
Heavy vocal load
High professional vocal demands
Speaking in large rooms
Speaking above background noise
Working in an air-conditioned room
Speaking in rooms with insufficient humidity
Working in an environment with smoke; essential goods

\begin{tabular}{|c|c|c|c|c|c|}
\hline \multicolumn{2}{|c|}{ Never } & \multicolumn{2}{|c|}{ Sometimes } & \multicolumn{2}{|c|}{ Often } \\
\hline$n$ & $\%$ & 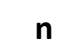 & $\%$ & $n$ & $\%$ \\
\hline 4 & 13.30 & 15 & 50.00 & 11 & 267 \\
\hline 4 & & 26 & & 0 & \\
\hline 10 & 0 & 20 & b & 0 & 0 \\
\hline 4 & 13 & 21 & & 5 & 6.70 \\
\hline 23 & 19. & 6 & 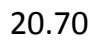 & 0 & 0.00 \\
\hline 5 & 16.70 & 20 & & 5 & 16.70 \\
\hline 20 & 83.30 & 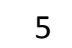 & 16.70 & 0 & 0.00 \\
\hline 2 & 70 & 23 & 0 & 5 & 16.70 \\
\hline 13 & 43.30 & 15 & 50.00 & 2 & 5.70 \\
\hline 17 & 56.70 & 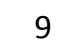 & 0 & 4 & 13.30 \\
\hline 7 & 23.30 & 18 & 60.00 & 5 & 16.70 \\
\hline 21 & 70.00 & . & 0 & 0 & 0.00 \\
\hline 4 & 13.30 & 16 & 30 & 10 & 33.30 \\
\hline 16 & 53.30 & 13 & 43.30 & 1 & 3.30 \\
\hline 13 & 43.30 & 12 & 40.00 & $J$ & 16.70 \\
\hline 2 & 6.70 & 5 & 16.70 & 23 & 76.70 \\
\hline 8 & 27.60 & 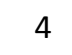 & 13. & 17 & 58.60 \\
\hline 1 & 3.30 & 12 & 40 & 17 & 56.70 \\
\hline 0 & 0.00 & 11 & 36. & 19 & 63.30 \\
\hline 14 & 48.30 & 12 & 41.40 & 3 & 10.30 \\
\hline 6 & 20.70 & 18 & 62.10 & 5 & 17.20 \\
\hline 20 & 66.70 & 8 & 26.70 & 2 & 6.70 \\
\hline
\end{tabular}




\section{Videolaryngostroboscopic evaluation}

The results of the videolaryngostroboscopic evaluation are presented in Table 5. In $61 \%$ ( $n=19$ of 31 ) of the subjects, glottic closure was complete during phonation. Forty-five percent $(n=14$ of 31 ) presented with an organic lesion. The most common organic lesion was erythema $(26 \%, n=8$ of 31$)$. The results of the observations of supraglottic contraction $M-L$ and A-P using the SERF protocol are presented in Table 6 . In the majority of the students, a certain degree of supraglottic M-L $(51.6 \%, n=16$ of 31$)$ and A-P $(67.7 \%, n=21$ of $31)$ constriction was found. Concordance value of the evaluations of the two voice therapists was $82.9 \%$.

Table 5. Results of the Videolaryngostroboscopy in Musical Theater Students

Evaluation of Vocal Folds

\begin{tabular}{|c|c|c|c|}
\hline \multirow{3}{*}{ Symmetry } & Symmetrical & 31 & 100.0 \\
\hline & Asymmetrical & 0 & 0.0 \\
\hline & Regular & 28 & 90.3 \\
\hline \multirow[t]{3}{*}{ Regularity } & Irregular & 1 & 3.2 \\
\hline & Inconsistent & 1 & 3.2 \\
\hline & Complete & 19 & 61.3 \\
\hline \multirow[t]{6}{*}{ Glottic closure } & Incomplete & 10 & 32.3 \\
\hline & Inconsistent & 1 & 3.2 \\
\hline & Normal & 19 & 61.3 \\
\hline & Longitudinal & 0 & 0.0 \\
\hline & Posterior & 7 & 22.6 \\
\hline & Anterior & 1 & 3.2 \\
\hline \multirow[t]{6}{*}{ Type of gap } & Irregular & 0 & 0.0 \\
\hline & Oval & 0 & 0.0 \\
\hline & Hourglass & 2 & 6.5 \\
\hline & Oval & 0 & 0.0 \\
\hline & Inconsistent & 1 & 3.2 \\
\hline & Normal & 29 & 93.5 \\
\hline \multirow{4}{*}{ Amplitude } & Increased & 0 & 0.0 \\
\hline & Reduced & 0 & 0.0 \\
\hline & None & 0 & 0.0 \\
\hline & Normal & 29 & 93.5 \\
\hline \multirow{3}{*}{ Mucosal wave } & Increased & 0 & 0.0 \\
\hline & Reduced & 0 & 0.0 \\
\hline & None & 0 & 0.0 \\
\hline \multirow{2}{*}{ Aspect of the vocal folds } & Normal & 17 & 54.8 \\
\hline & Organic lesion & 14 & 45.2 \\
\hline \multirow{4}{*}{ Type of lesion } & Nodules & 3 & 9.7 \\
\hline & Erythema & 8 & 25.8 \\
\hline & Edema & 2 & 6.5 \\
\hline & Polyp & 1 & 3.2 \\
\hline
\end{tabular}

$\%$

0.3

61.3

1.3

0.0

2.6

3.2

0.0

3.5

0

0.0

93.5

0

4.8

2

9.7

5.8

3.2 
Table 6. Evaluation of the Supraglottic Constriction Using the SERF Protocol in Musical Theater Students

Evaluation of M-L and A-P Constriction during Phonation Mean Median SD Minimum Maximum $M-L$ constriction

$\begin{array}{ccccc}0.55 & 1 & 0.57 & 0 & 2 \\ 1.1 & 1 & 1.01 & 0 & 4\end{array}$

A-P constriction

Abbreviations: A-P, anterior-posterior; M-L, mediolateral.

\section{Perceptual evaluation}

The median scores of the GRBASI parameters for males and females were 0 for $\mathrm{G}$ (range: $0-$ 1, mean: 0.23 , SD: 0.43 ), 0 for $R$ (range: $0-1$, mean: 0.13 , SD: 0.34 ), 0 for $B$ (range: $0-1$, mean: 0.23, SD:0.43), 0 for A (range: 0-0, mean: 0, SD: 0), 0 for S (range: 0-0, mean: 0, SD: 0 ), and 0 for I (range: $0-0$, mean: 0, SD: 0 ). Four voices ( $n=4$ of $31,13 \%$ ) were evaluated as mildly rough and seven voices were evaluated as mildly breathy ( $n=7$ of $31,22.5 \%$ ). Concordance value of the perceptual ratings of the two voice therapists was $92.8 \%$.

Table 7. Results of the Objective Voice Analysis in Musical Theater Students

Men Women

Mean Median Minimum Maximum SD Mean Median Minimum Maximum SD Aerodynamic measurements

\begin{tabular}{|c|c|c|c|c|c|c|c|c|c|c|}
\hline MPT & 22.2 & 23.8 & 9.1 & 31.8 & 7.2 & 18.7 & 18.5 & 10.3 & 29.8 & 4.5 \\
\hline VC & 3814.3 & 3700.0 & 3400.0 & 4600.0 & 414.0 & 2375.0 & 2275.0 & 1900.0 & 3200.0 & 333.0 \\
\hline$P Q$ & 194.0 & 164.6 & 113.1 & 373.6 & 85.3 & 132.8 & 128.9 & 73.9 & 204.7 & 32.5 \\
\hline \multicolumn{11}{|c|}{ Voice Range Profile } \\
\hline llow & 57.0 & 57.0 & 50.0 & 62.0 & 4.4 & 55.4 & 55.5 & 50.0 & 61.0 & 3.1 \\
\hline Ihigh & 109.9 & 109.0 & 104.0 & 118.0 & 5.1 & 109.1 & 109.0 & 95.0 & 118.0 & 6.4 \\
\hline Flow & 75.6 & 77.8 & 65.7 & 87.3 & 7.0 & 138.3 & 138.6 & 71.0 & 164.8 & 18.3 \\
\hline Fhigh & 781.8 & 830.6 & 523.3 & 1046.5 & 227.8 & 1208.9 & 1174.7 & 740.0 & 1661.8 & \\
\hline \multicolumn{11}{|c|}{ Acoustic analysis } \\
\hline $\mathrm{F}_{0}$ & 126.3 & 126.4 & 108.2 & 153.0 & 14.0 & 218.9 & 216.8 & 185.8 & 270.1 & \\
\hline Jitter & 0.64 & 0.52 & 0.26 & 1.46 & 0.40 & 1.01 & 0.78 & 0.26 & 2.59 & 0.66 \\
\hline Shimmer & 3.28 & 3.70 & 1.89 & 4.41 & 0.94 & 3.54 & 3.42 & 2.27 & 5.91 & $0 . \varepsilon$ \\
\hline $\mathrm{vF}_{0}$ & 0.90 & 0.86 & 0.61 & 1.37 & 0.27 & 1.06 & 0.97 & 0.43 & 2.15 & 0.4 \\
\hline NHR & 0.14 & 0.14 & 0.13 & 0.15 & 0.01 & 0.12 & 0.12 & 0.10 & 0.17 & 0.0 \\
\hline & 3.9 & 5.3 & -1.0 & 6.2 & 2.7 & 5.6 & 5.7 & 0.6 & 10.4 & \\
\hline
\end{tabular}

Abbreviations: DSI, Dysphonia Severity Index; $F_{0}$, mean fundamental frequency; MPT, maximal phonation time; NHR, noise-to-harmonic ratio; $\mathrm{PQ}$, phonation quotient; $\mathrm{VC}$, vital capacity; $\mathrm{vF}_{0}$, variation of $F_{0}$. 


\section{Objective voice assessment}

The results of the objective voice assessment are presented in Table 7. The median DSI scores of the male and female musical students are, respectively, +5.3 and +5.7 , both corresponding with a very good vocal quality. The DSI values vary between -1 and +10.4 . Only $1 \%$ of the subjects ( $n=3$ of 31 ) had a vocal quality below the cutting point of 1.6.

\section{Comparison with the follow-up assessment}

Seven students ( 2 males and 5 females) agreed to participate in a follow-up study 1 year after the first assessment. Results of the comparison between the first and the follow-up assessments of the objective voice analysis and the videolaryngostroboscopic evaluation are presented in Table 8. No statistically significant differences in the voice parameters between the first and the second assessments were found.

Table 8. Comparison of the Voice Data Between the First Assessment and a Follow-Up Assessment 1 Year Later

\section{Measurement}

Objective voice assessment

Aerodynamic measurements

MPT

VC

$P Q$

Voice Range Profile

llow

Ihigh

Flow

Fhigh

Acoustic Analysis

$\mathrm{F}_{0}$

Jitter

Shimmer

$\mathrm{vF}_{0}$

NHR

DSI

Questionnaires

VHI

$\mathrm{VHI}$ singing voice

Videolaryngostroboscopic evaluation

Symmetry

Regularity

Glottic closure

Type of gap

Amplitude

Mucosal wave

Aspect of the vocal folds

\section{$P$ Value}

0.063

0.246

0.499

0.107

0.400

0.753

0.917

0.735

0.310

0.128

1.000

0.932

0.735

0.715

0.446

0.184

0.781

0.848

0.901

Variable is constant

Variable is constant

0.202 


$\begin{array}{lc}\text { Measurement } & \boldsymbol{P} \text { Value } \\ \text { Type of lesion } & 0.834 \\ \text { M-L constriction } & 1.000 \\ \text { A-P constriction } & 0.083\end{array}$

\begin{abstract}
Abbreviations: A-P, anterior-posterior; DSI, Dysphonia Severity Index; $F_{0}$, mean fundamental frequency; M-L, mediolateral; MPT, maximal phonation time; NHR, noise-to-harmonic ratio; $P Q$, phonation quotient; $\mathrm{VC}$, vital capacity; $\mathrm{VF}_{0}$, variation of $\mathrm{F}_{0}$; $\mathrm{VHI}$, Voice Handicap Index.
\end{abstract}

\title{
Discussion
}

The main purpose of this study was to investigate the vocal fold status and the vocal quality of the speaking voice, voice symptoms, and vocal habits in 31 musical theater students using a combination of subjective and objective voice measurements. The median DSI scores of the male and female students were +5.3 and +5.7 , reflecting a very good vocal quality. The DSI values vary between -1 and +10.4 . Only $1 \%$ of the subjects $(n=3$ of 31 ) had a vocal quality below the cutoff point of 1.6. Musical theater students is very important because they are absolutely depending on their vocal quality and vocal capacities for their studies and their future profession. The median DSI of the group of musical theater students is 5.5 corresponding with a DSI\% of more than $100 \%$ (105\%). The objective vocal quality measured with the DSI in this group of musical theater students is better than the normative data of the Belgian Study Group on Voice Disorders. ${ }^{21}$ Compared to students of the master program speech language therapy having a borderline vocal quality (DSI of 68\%), the objective vocal quality of musical theater students is much better. ${ }^{22}$ These findings support the hypothesis that vocal capacities in this specific group of future elite vocal professionals need to be optimal to manage the heavy vocal demands. Timmermans et $\mathrm{al}^{6}$ investigated the vocal quality of future stage and musical actors using the DSI and found a rather poor vocal quality compared to future nonvocal professionals. The DSI of the future actors in the study of Timmermans et $\mathrm{al}^{6}$ was 2.3 or $73 \%$, which is $32 \%$ less than the DSI in this study. The different results are possibly due to the different inclusion criteria of the actors. In the study of Timmermans et al, $^{6}$ a combination of stage and musical actors was included. To the best of our knowledge, there are no other studies investigating musical theater students using the multiparameter index DSI. When a musical theater student is consulting a voice clinic, it is important that clinicians dispose of normative data adjusted to this specific type of voice user. The database of this study with the results of the objective voice assessment consisting of aerodynamic measurements, acoustic analysis, voice range, and DSI can be used as a reference for further studies and to compare the vocal characteristics of new musical theater students.

For musical theater actors, a perceptual normal voice is an important criterion so that they are maximally intelligible, able to project their voice, and able to perform their singing tasks. In this study, the speaking voices were perceptually evaluated as normal. The mean score for grade of dysphonia (G-score) of the GRBASI scale was 0.24 (median of 0 ). In the study of Timmermans et $\mathrm{al}^{6}$, future stage and musical actors had a slightly higher mean perceptual G- 
score of 0.64 . The results of the perceptual evaluation of the voices in this study were in accordance with the overall good objective vocal quality of the subjects.

As described in the introduction, environmental factors play an important role in vocal performance. Another goal of this study was to describe the vocal behavior and the risk factors for developing voice problems in this population. Timmermans et al ${ }^{6}$ attributed the poor vocal quality of the future elite vocalperformers to poor vocal hygiene. Donahue et $\mathrm{al}^{7}$ investigated the vocal symptoms and habits of musical theater students and found that the majority noted poor hydration habits and more than $50 \%$ of the students reported at least one current negative vocal symptom. Donahue et $\mathrm{al}^{7}$ also recommended further education regarding vocal hygiene in the group of musical theater students. In this study, voice symptoms and risk factors for developing voice problems were evaluated using two checklists. ${ }^{12 ; 13}$ The impact of voice problems regarding the speaking and the singing voice on the psychosocial functioning was investigated using, respectively, the VHI and the VHI adapted to the singing voice. Although the musical theater students had higher scores on the $\mathrm{VHI}$ adapted to the singing voice compared to the $\mathrm{VHI}$, the median scores for both $\mathrm{VHI}$ scales were below the cutting point of a clinical score (less than 20). The questionnaires regarding voice symptoms revealed that voice symptoms are frequently reported on a regular basis (daily or weekly). The most frequently reported symptoms are vocal fatigue (80\%), dryness of the throat (80\%), and vocal tract discomfort (73.3\%). Thirty-seven percent of the musical theater students experience vocal fatigue on a regular basis (daily or weekly). The prevalence of vocal fatigue in this study is even higher than that reported by Phyland et $\mathrm{al}^{5}\left(\mathrm{n}=8\right.$ of $43,18.6 \%$ ) and Donahue et $\mathrm{al}^{7}(27.7 \%)$. Other frequently reported symptoms are irritation or pain (53.3\%), tickling feeling of the throat (53.3\%), and the feeling of throat tightness and pressure in the throat (41\%). The symptoms of a dry, tickling, painful, and irritable feeling in the throat all refer to a type of vocal tract discomfort and should be investigated in the future using the Vocal Tract Discomfort Scale. ${ }^{23}$ The high prevalence of vocal fatigue and vocal tract discomfort can possibly be attributed to the high vocal demands in this group. The high prevalence of voice symptoms in musical theater students is in contrast with the overall good objective vocal quality measured with the DSI.

In the literature, the heavy vocal load in musical theater actors is already described. ${ }^{5}$ This study provides an overview of the occurrence and the amount of vocal misuse in the musical theater students. The participants in this study report harmful vocal habits like screaming (70\%), habitual loud voice use (47.3\%), speaking with a tensed voice $(56.7 \%)$, frequently coughing $(43.3 \%)$ or throat clearing $(53,7 \%)$, and shouting above background noise (66.7\%). The most notable characteristic was the high prevalence of anxiety or stress (86.7\%). When studying the amount of vocal misuse, most behaviors occur on a monthly basis. In further research, the amount of vocal misuse should be compared to other groups of students. Some of the reported vocal misuse is inherent to the speech tasks as an actor (screaming, habitual loud voice, speaking with tensed voice). Roy et $\mathrm{al}^{4}$ described this as vocally violent behavior in actors. To limit potential damage, techniques can be learned to use the voice in a more hygienic, less abusive manner. ${ }^{4}$ The techniques are summarized by Roy et $\mathrm{al}^{4}$ and focus on (a combination of) "muscular release, an open pharynx and jaw, abdominal breath support, focus of tone, placement of pitch, absence of glottal attack, extension of vowels, and a warm-up" (p. 216). However, further research is necessary to investigate the effectiveness of these techniques in preventing vocal injuries. Other 
reported vocal misuse like throat clearing, smoking, and coughing are not inherent to the profession of actor and should be addressed during voice counseling sessions in the program. Donahue et $\mathrm{al}^{7}$ also recommended further education regarding vocal hygiene in the group of musical theater students.

A striking observation in this study is the high presence of organic lesions of the vocal folds in this group of musical theater students despite their overall good objective and perceptual vocal quality. In $45 \%$ of the students, videolaryngostroboscopic evaluation of the vocal folds showed an organic lesion. More male than female students showed an organic lesion (86\% versus $33 \%)$. The majority of these lesions are inflammatory lesions (26\%). These results are in accordance with Timmermans et al $^{6}$ showing the same prevalence $(27 \%)$ of inflammatory lesions in future elite vocal performers. Because musical theater students are absolutely depending on their vocal quality and vocal capacities for their future profession and studies, these results emphasize the need for a specialized medical voice screening at the beginning of the program, with an accurate and frequent follow-up during the whole program.

Whether the number of lesions is significantly higher in male students compared to female students is a question for further research in larger study groups. Another observation from the videolaryngostroboscopic assessment of the vocal folds is the presence of supraglottic constriction during habitual phonation. The median supraglottic activity in M-L and A-P directions using the 4-point grading scale of the SERF protocol was grade 1 (mean: respectively 0.6 and 1.1 ) varying from 0 to 2 . Supraglottic compressions have commonly been described as signs of vocal hyperfunction. ${ }^{24}$ In the recent literature, however, supraglottic constriction has also been found in healthy singers. ${ }^{25 ; 26 ; 27}$ Healthy opera, ${ }^{27}$ pop, rock, and jazz singers ${ }^{25 ;}{ }^{26}$ showed $\mathrm{M}-\mathrm{L}$ and especially A-P supraglottic activity during singing. In rock singers, ${ }^{25}$ the supraglottic activity was found in the singing as well as the speaking voice. These authors ${ }^{25}$ suggest that supraglottic activity during professional voice use may not always be a hyperfunctional behavior. In this study, it is not clear whether the supraglottic behavior is a sign of hyperfunction or a speaking or singing strategy to increase projection. Further research to investigate the supraglottic activity in musical theater actors during singing and speaking is necessary. Laryngeal abnormalities occur commonly in asymptomatic professional voice users. ${ }^{28 ;} 29$ Caution is needed when interpreting the relationship between an observed laryngeal abnormality and a patient's voice complaint. ${ }^{28 ;}{ }^{29}$ Elias et al $^{29}$ recommended baseline examinations of professional voice users when they are healthy and asymptomatic to establish each individual's "normal" condition. The results of this study emphasize the importance of a baseline examination in musical theater students, because vocal pathology does not always correlate with voice quality.

In a second purpose, the vocal quality of seven students was reevaluated 1 year after the first assessment. An improvement in vocal quality and a decrease of vocal pathology were hypothesized due to the singing training. However, no differences were found in objective or subjective vocal quality after 1 year. The heavy vocal load, the presence of harmful vocal habits, anxiety and stress, the lack of vocal hygiene, and medical follow up are possible causes for the lack of improvement in vocal pathology Whether the integration of a vocal hygiene program is beneficial for the vocal quality is subject for further research. The number of subjects included in the follow-up study was small. To investigate the evolution of the vocal quality during the program, further research is necessary. 


\section{Conclusion}

Musical theater students show an overall excellent vocal quality. The mean DSI scores in male and female musical theater students were, respectively, 5.3 and 5.7, both corresponding with good vocal capacities. Although the musical theater students had higher scores on the $\mathrm{VHI}$ adapted to the singing voice compared to the $\mathrm{VHI}$, the median scores for both VHI scales were below the cutting point of a clinical score (less than 20). However, questionnaires investigating voice symptoms and vocal behavior revealed the presence of vocal fatigue, dryness of the throat, vocal tract discomfort, and harmful vocal habits in the majority of students. Despite the overall good vocal quality, there is a high presence of inflammatory lesions of the vocal folds in this group of musical theater students. ENT specialists must be aware of the presence of these vocal fold disorders. A follow-up voice assessment in seven students showed no evolution in the vocal quality and the vocal fold status. Because musical theater students are absolutely depending on their vocal quality and vocal capacities for their future profession and studies, the results of this study emphasize the need for a profound medical voice screening at the beginning of the program with an accurate follow-up during the whole program.

\section{References}

1. Koufman JA, Isaacson G. Voice Disorders. Philadelphia, PA: WB Saunders; 1999.

2. Hoffman-Ruddy B, Lehman J, Crandell C, et al. Laryngostroboscopic, acoustic, and environmental characteristics of high-risk vocal performers. J Voice. 2001;15:543-552.

3. Raphael BN, Scherer RC. Voice modifications of stage actors: acoustic analyses. J Voice. 1987;1:83-87.

4. Roy N, Ryker KS, Bless DM. Vocal violence in actors: an investigation into its acoustic consequences and the effects of hygienic laryngeal release training. J Voice. 2000;14:215230.

5. Phyland DJ, Thibeault SL, Benninger MS, et al. Perspectives on the impact on vocal function of heavy vocal load among working professional music theater performers. $J$ Voice. 2013;27:390, e31-e39.

6. Timmermans B, De Bodt MS, Wuyts FL, et al. Poor voice quality in future elite vocal performers and professional voice users. J Voice. 2002;16:372-382.

7. Donahue EN, Leborgne WD, Brehm SB, et al. Reported vocal habits of first-year undergraduate musical theater majors in a preprofessional training program: a 10-year retrospective study. J Voice. 2014;28:316-323.

8. De Bodt M, Jacobson B, Musschoot S, et al. The Voice Handicap Index: an instrument for the quantification of psychosocial consequences of voice disorders. Logopedie. 2000;13:29-33.

9. Jacobson BH, Johnson A, Grywalski C, et al. The Voice Handicap Index (VHI): development and validation. Am J Speech Lang Pathol. 1997;6:66-70.

10. D'haeseleer A, Morsomme D, De Bodt M. Ontwikkeling, standaardisatie en validatie van de "VHI aangepast aan de zangstem". Logopedie. 2011;4:72-80.

11. Morsomme D, Gaspar M, Jamart J, et al. Adaption du Voice Handicap Index a la voix chantee. Rev Laryngol Otol Rhinol (Bord). 2007;128:305-314.

12. De Bodt M, Mertens F, Heylen L. Werken aan stem. Antwerp: Garant; 2008.

13. Russel A, Oates J, Pemberton C. Voice Care for Teachers. Flinders University; 2000.

14. Remacle M. The contribution of videolaryngostroboscopy in daily ENT practice. Acta Otorhinolaryngol Belg. 1996;50:265-283.

15. Poburka BJ. A new stroboscopy rating form. J Voice. 1999;13:403-413. 
16. Hirano M. Clinical Examination of Voice. New York, NY: Springer Verlag; 1981.

17. Dejonckere PH, Remacle M, Fresnel-Elbaz M, et al. Differential perceptual evaluation of pathological voice quality: reliability and correlations with acoustic measurements. Rev Laryngol Otol Rhinol (Bord). 1996;117:219-224.

18. Heylen L, Wuyts FL, Mertens F, et al. Evaluation of the vocal performance of children using a voice range profile index. J Speech Lang Hear Res. 1998;41:232-238.

19. Wuyts FL, De Bodt MS, Molenberghs G, et al. The Dysphonia Severity Index: an objective measure of vocal quality based on a multi-parameter approach. J Speech Lang Hear Res. 2000;43:796-809.

20. Raes J, Wuyts F, De Bodt M, et al. The Dysphonia Severity Index used with a percentage scale. Stem-, Spraak- en Taalpathologie. 2002;11:30-37.

21. Van De Heyning P, Remacle $M$, Van Cauwenberge $P$, et al. Research work of the Belgian Study Group on voice disorders. Acta Otorhinolaryngol Belg. 1996;50:321-386.

22. Van Lierde KM, D'haeseleer E, Wuyts F, et al. The objective vocal quality, vocal risk factors, vocal complaints and corporal pain in Dutch female students training to be speech language pathologists during the four years of study. J Voice. 2010;24:592-598.

23. Luyten A, Bruneel L, Meerschman I, et al. Prevalence of vocal tract discomfort in the Flemish population without self-perceived voice disorders. J Voice. 2016;30:308-314.

24. Stager S, Bielamowicz S, Regnell J, et al. Supraglottic activity: evidence of hyperfunction or laryngeal articulation? J Speech Hear Res. 2000;43:229-238.

25. Guzman M, Barros M, Espinoza F, et al. Laryngoscopic, acoustic, perceptual, and functional assessment of voice in rock singers. Folia Phoniatr Logop. 2013;65:248-256.

26. Guzman M, Lanas A, Olavarria C, et al. Laryngoscopic and spectral analysis of laryngeal and pharyngeal configuration in non-classical singing styles. J Voice. 2015;29:130, e21-e28.

27. Mayerhoff RM, Guzman M, Jackson-Menaldi C, et al. Analysis of supraglottic activity during vocalization in healthy singers. Laryngoscope. 2014;124:504-509.

28. Sataloff RT, Hawkshaw MJ, Johnson JL, et al. Prevalence of abnormal laryngeal findings in healthy singing teachers. J Voice. 2012;26:577-583.

29. Elias ME, Sataloff RT, Rosen DC, et al. Normal strobovideolaryngoscopy: variability in healthy singers. J Voice. 1997;11:104-107. 